Relations industrielles

Industrial Relations

\title{
Cousineau, Jean-Michel, Économie du travail, 2e éd.
}

\section{Ross Finnie}

Volume 48, numéro 1, 1993

URI : https://id.erudit.org/iderudit/050845ar

DOI : https://doi.org/10.7202/050845ar

Aller au sommaire du numéro

Éditeur(s)

Département des relations industrielles de l'Université Laval

ISSN

0034-379X (imprimé)

1703-8138 (numérique)

Découvrir la revue

Citer ce compte rendu

Finnie, R. (1993). Compte rendu de [Cousineau, Jean-Michel, Économie du travail, 2e éd.] Relations industrielles / Industrial Relations, 48(1), 203-205.

https://doi.org/10.7202/050845ar

Tous droits réservés (C) Département des relations industrielles de l'Université Laval, 1993
Ce document est protégé par la loi sur le droit d'auteur. L'utilisation des services d'Érudit (y compris la reproduction) est assujettie à sa politique d'utilisation que vous pouvez consulter en ligne.

https://apropos.erudit.org/fr/usagers/politique-dutilisation/ 
Économie du travail, $2^{\circ}$ édition, par Jean-Michel Cousineau, Boucherville, Qué., Gaëtan Morin, éditeur, 1989, 248 p., ISBN 2-89105-311-7

À l'endos de cet ouvrage, il est écrit: "Ce livre rend compte des réponses économiques en matière de formation de l'emploi et des salaires dans une économie de marché, au sujet du rôle et de l'effet du syndicalisme sur le marché du travail et des raisons et des limites de l'intervention de l'État... Compte tenu de l'expérience et de l'expertise de l'auteur, l'accent est placé autant que possible sur les théories pour lesquelles les éléments de preuve sur le plan empirique sont abondants ». Ce livre comporte trois parties. La première occupe plus de la moitié de l'ouvrage et s'intitule : "La formation de l'emploi et des salaires dans une économie de marché ». La deuxième "Le syndicalisme et la négociation collective " comprend 40 pages et la troisième aborde un sujet peu traité dans les ouvrages en économie du travail « L'intervention de l'État, l'intérêt général et les décisions publiques ", elle comprend 54 pages. Par la suite, il y a une dizaine de pages de références qui correspondent aux chapitres et, finalement, un index de 4 pages sur les termes utilisés et un index des auteurs auxquels l'auteur réfère.

Dans la première partie, l'auteur aborde des sujets classiques en économie du travail. Au chapitre 1, il débute par une vue d'ensemble de l'offre, de la demande, de l'équilibre et des dynamiques du marché du travail (16 pages), comprenant quelques tableaux des salaires selon l'industrie, l'occupation et la région. Il poursuit avec des chapitres sur "La demande de travail et la détermination de l'emploi " (36 pages); "L'interaction de l'offre et de la demande et la détermination des salaires " ( 9 pages); " La théorie du capital humain » ( 9 pages); «Les disparités salariales entre les hommes et les femmes » (7 pages); "L'effet syndical " sur les salaires (3 pages); "La théorie hédonique des salaires " qui consiste en une discussion des salaires compensatoires (9 pages); "L'évolution des salaires à travers le temps " (23 pages), et une brève "Conclusion " (4 pages).

L'auteur développe ces sujets avec un accent sur les fondements de la théorie microéconomique. La présentation de la matière est rigoureuse et minutieuse, sans toutefois être alourdie par le côté technique, ce qui constitue un équilibre délicat que l'auteur a bien réussi à atteindre. Les graphiques sont clairs, utiles et bien intégrés au texte. La présentation générale de ces chapitres est très claire et ils nous fournissent une base solide de la théorie microéconomique, des principes-clés du marché du travail. Par exemple, en présentant la théorie de la demande de travail dans les chapitres 2 et 3 , l'auteur développe en détail la théorie (néoclassique) de production de l'entreprise et, par conséquent, situe bien la demande de travail dans un contexte très précis selon la théorie microéconomique.

Par contre, dans cette première partie, qui est la plus importante de l'ouvrage, je note un certain déséquilibre. Par exemple, l'offre de travail, dont l'analyse occupe une place centrale dans d'autres ouvrages de ce genre, n'est traitée que dans une dizaine de pages seulement, en annexe du chapitre 2 , sous le titre " La détermination des heures de travail ". Il n'y a également aucune discussion sur les politiques économiques qui impliquent d'une façon importante l'offre de travail, telles l'aide sociale, l'assurancechômage, etc. Aussi, le traitement de l'économie de la famille ne comporte qu'une seule page à la fin de cette annexe sur l'offre de travail. Finalement, l'analyse de l'équilibre sur le marché du travail comme telle ne constitue qu'une seule page, en fin du chapitre 
3, intitulée « L'interaction de l'offre et de la demande et la détermination des salaires » (où ce traitement est implicite plutôt qu'explicite, c'est-à-dire l'offre de travail est considérée comme un des facteurs qui déterminent la valeur de la productivité marginale, parallèle au ratio capital-travail et à la demande pour le produit). Il semble que l'accent soit constamment mis sur la demande de travail dérivée du choix de production de l'entreprise, et qu'il ne soit pas suffisamment axé sur le choix des individus du côté de l'offre de travail. Une étudiante, de qui j'ai sollicité des commentaires, a mentionné : « J'ai eu l'impression de lire la seconde partie d'un livre de microéconomique, soit l'entreprise et sa production, plutôt qu'un livre d'économie du travail. » Il est donc possible que le lecteur termine cette section sans assez d'appréciation de la détermination des salaires sur le marché du travail selon les courbes (indépendantes!) de la demande et de l'offre de travail. En général, $\mathrm{j}$ 'aurais préféré voir un traitement plus élaboré de l'offre de travail pour aborder des questions importantes qui impliquent ce développement et pour rendre le développement du marché du travail plus équilibré.

De plus, ce livre ne présente que très peu de données empiriques décrivant la situation actuelle ou l'évolution historique du travail au Canada. Par exemple, il faut consulter d'autres ouvrages pour connaître les taux de participation sur le marché du travail ou les tendances du nombre d'heures travaillées. Dans le chapitre où il est question de l'effet syndical, on ne présente pas la force de cet effet au Canada ou ailleurs, ni à travers le temps. Également, en présentant les disparités salariales entre les hommes et les femmes, $\mathrm{j}$ 'aurais aimé voir plus de données concernant l'écart salarial; par exemple, l'évolution dans le temps ou les différences selon l'occupation, l'industrie, l'âge, etc., plutôt que le simple fait rapporté que « l'écart salarial entre les hommes et les femmes se situe en moyenne à $40 \%$ » (p. 77). Dans le chapitre traitant du capital humain, il n'est pas fait mention des différences des salaires selon le niveau de scolarité. En général, cet ouvrage ne discute guère des travaux empiriques, ni de résultats, ni de méthodologie, ni de simples chiffres décrivant généralement les sujets abordés tels que précédemment mentionnés. Des références aux travaux empiriques apparaissent à la fin du livre, mais elles ne sont pas intégrées au texte. Ainsi, un professeur qui utilise cet ouvrage pour guider ses étudiants aimerait probablement ajouter les statistiques simples et les exemples des travaux empiriques comme supplément important au bon développement de la théorie que l'auteur nous présente.

En ce qui concerne l'organisation des chapitres, $j$ 'ai trouvé quelques aspects un peu curieux. J'ai déjà mentionné que le sujet de l'offre de travail apparaît seulement dans une annexe au chapitre sur la demande de travail, mais il est encore plus étonnant de retrouver le développement sur les cas monopsone et monopole dans l'annexe du chapitre sur les disparités salariales entre les hommes et les femmes, sans aucun lien particulier avec la première partie du chapitre. De plus, ce sujet secondaire prend autant de place que le sujet supposément principal de ce chapitre, nous laissant seulement 7 pages pour la présentation des disparités. Finalement, il y a des applications (i.e. questions) au début du livre dans deux chapitres, et plus rien après.

La seconde partie aborde les syndicats et la négociation collective et comporte cinq courts chapitres : "Les objectifs économiques des syndicats " (8 pages); "Le rôle des syndicats » et le modèle exit-voice (4 pages); «Le pouvoir syndical », sur les conditions classiques de cette question (10 pages); "L'activité de grève " (10 pages), qui 
traite des modèles de Rees, de Hicks, et de Siebert et Addison; et « L'évolution prévisible du syndicalisme » (8 pages). De plus, tel que mentionné déjà, il y a également, au chapitre 6 de la première section, trois courtes pages qui introduisent le concept du pouvoir monopolistique des syndicats. (On ne comprend pas pourquoi cette petite section ne se trouve pas dans la deuxième section du livre.) En général, le traitement est semblable à la première partie, c'est-à-dire bien structuré en regard de la théorie microéconomique, mais on ne retrouve pas beaucoup de discussions sur les institutions, peu de chiffres sur les tendances de la situation réelle à travers le temps, ni aucune présentation des travaux empiriques dans ce domaine. Par exemple, le lecteur ne parvient pas à saisir le taux de syndicalisation au Canada en général, ni comment il a changé à travers le temps. L'auteur développe bien la théorie du pouvoir des syndicats, mais il ne mentionne ni les chiffres spécifiques décrivant les effets salariaux ni la littérature énorme qui aborde les questions d'estimation de ces effets. L'auteur présente clairement les théories de grèves, mais il omet toutefois de donner leur fréquence ou leur tendance dans le temps. Ainsi, les théories manquent de précision, et un professeur préférerait probablement utiliser ces chapitres avec d'autres références qui établiraient le lien entre la théorie et la réalité.

La troisième partie, qui traite de « L'intervention de l'État, l'intérêt général et les décisions publiques ", ne se retrouve normalement pas dans un ouvrage sur l'économie du travail. Elle consiste principalement en une présenttion assez détaillée des principes microéconomiques, de l'équilibre général et des conditions requises pour obtenir un équilibre optimal global ainsi que les raisons de l'intervention de l'État. L'auteur termine avec un traitement de la théorie des choix publics. Plus précisément, cette section comprend les chapitres suivants : «L'optimum économique » (9 pages); « Les principaux théorèmes de l'économie du bien-être " (12 pages); " Les raisons de l'intervention de l'État » (22 pages); et « Les choix publics » (8 pages). La présentation est claire et, dans l'ensemble, toujours très bien fondée sur les principes microéconomiques. Elle est assez technique et très intuitive à la fois - ce qui est diffícile à réaliser. Il est un peu étonnant que ces sujets occupent une place si importante dans cet ouvrage, et qu'il n'y ait pas de lien particulièrement évident entre cette section et l'économie du travail comme telle. Par exemple, à la suite de la présentation de la théorie de l'équilibre général, l'auteur considère, dans un contexte très général, les problèmes tels les biens publics, les effets externes, les coûts décroissants et les risques et incertitudes, de même que le choix public; aucune de ces questions n'est traitée dans le contexte spécifique de l'économie du travail. Cette section pourrait donc servir pour un cours en économie publique.

En conclusion, Économie du travail, par Jean-Michel Cousineau, est un ouvrage qui occupe une place spéciale et unique parmi les ouvrages de ce genre, son approche est toujours fortement basée sur la théorie microéconomique et ses présentations sont complètes, rigoureuses, et claires. Par contre, si un professeur voulait utiliser ce manuel, il préférerait peut-être faire ses propres liens entre cette excellente base théorique et les chiffres, les institutions, les politiques économiques importantes et les travaux empiriques en général. Enfin, cet ouvrage fournit des opportunités intéressantes pour un cours en économie du travail, en relations industrielles ou encore en économie publique. 